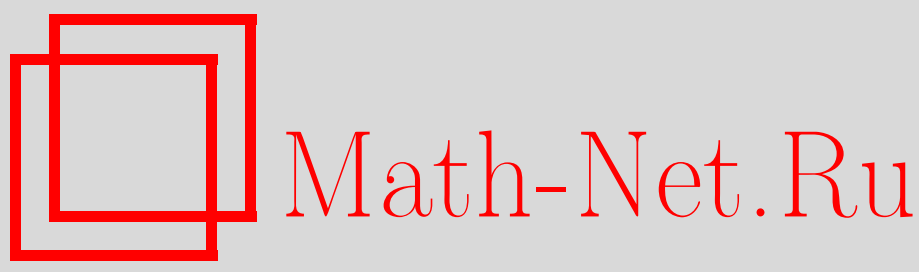

В. Р. Фаталов, Большие уклонения для гауссовских процессов в гёльдеровской норме, Изв. РАН. Сер. матем., 2003, том 67, выпуск 5, 207-224

DOI: https://doi.org/10.4213/im457

Использование Общероссийского математического портала Math-Net.Ru подразумевает, что вы прочитали и согласны с пользовательским соглашением

http://www. mathnet.ru/rus/agreement

Параметры загрузки:

IP : 52.6 .47 .48

26 апреля 2023 г., $17: 38: 57$ 
УДК 519.21

В.Р. Фаталов

\title{
Большие уклонения для гауссовских процессов в гёльдеровской норме
}

\begin{abstract}
Доказаны результаты о точных асимптотиках вероятностей больших уклонений для гауссовских процессов в гёльдеровской норме. Рассмотрены следующие классы процессов: винеровский процесс, броуновский мост, дробное броуновское движение, стационарные гауссовские процессы со степенной ковариационной функцией. Метод исследования - метод двойных сумм для гауссовских полей.

Библиография: 32 наименования.
\end{abstract}

\section{§1. Введение и формулировка основных результатов}

В работе [1] А.Н. Колмогоров предложил общий эффективный метод исследования непрерывности п.н. выборочных функций случайных процессов. Известно, что многие случайные процессы имеют непрерывные, но недифференцируемые траектории, поэтому изучение гёльдеровских свойств этих процессов приобретает важное значение. Метод, представленный в работе [1], был далее развит в рабо$\operatorname{tax}[2],[3]$, где были найдены удобные достаточные условия гёльдеровости траекторий гауссовских процессов. Современное состояние вопроса представлено в книге $[4, \S 4.4$ и гл. 9] (там использован эквивалентный термин "условие Липшица") и работе [5].

В настоящей статье для некоторых классов гауссовских процессов $\xi(t)$ вычислим точную асимптотику вероятности

$$
\mathrm{P}\left\{\|\xi\|_{\gamma}>u\right\}, \quad u \rightarrow \infty
$$

где $\|\cdot\|_{\gamma}$ - определяемая ниже, в формуле $(1.3)$, стандартная гёльдеровская норма на отрезке $[0,1], 0 \leqslant \gamma<1$.

Рассмотрим следуюшие классы процессов: винеровский процесс, броуновский мост, дробное броуновское движение и стационарные гауссовские процессы со степенной ковариационной функцией.

Отметим, что вычисление точной асимптотики вероятности (1.1) - это чрезвычайно сложная задача. Применяемый в настоящей статье подход к этой проблеме основан на результатах, полученных методом двойных сумм ранее в [6]-[10], где были найдены точные асимптотики вероятностей

$$
\mathrm{P}\left\{\sup _{t \in T}|X(t)|>u\right\}, \quad u \rightarrow \infty
$$

Работа выполнена при финансовой поддержке РФФИ (грант № 01-01-00649).

$$
\text { (C) В.Р. ФАТАЛОВ, } 2003
$$


для некоторого широкого класса гауссовских полей $X(t)$, заданных на компакте $T \subset \mathbb{R}^{k}, k \geqslant 1$.

Ранее точная асимптотика вероятности (1.1) при $0<\gamma<1$, по-видимому, не исследовалась. В работах [11]-[13] доказан принцип больших уклонений для винеровского процесса в гёльдеровской норме и получена точная асимптотика типа Шильдера для нормы, эквивалентной стандартной гёльдеровской (см. также [27]-[29]).

Перейдем к изложению результатов, полученньг в данной статье.

Пусть $C_{0}[0,1]$ - множество непрерывных на отрезке $[0,1]$ функций $x(t)$, равных нулю в нуле.

Для $0 \leqslant \gamma \leqslant 1$ определим функционал

$$
\|x\|_{\gamma}:=\sup _{t, s \in[0,1], t \neq s} \frac{|x(t)-x(s)|}{|t-s|^{\gamma}}, \quad x \in C_{0}[0,1] .
$$

Как известно, гёльдеровское пространство $\mathscr{C}_{\gamma}=\mathscr{C}_{\gamma}[0,1]$ порядка $\gamma$ определяется следующим образом:

$$
\mathscr{C}_{\gamma}=\left\{x \in C_{0}[0,1]:\|x\|_{\gamma}<\infty\right\}
$$

Отметим, что $\left(\mathscr{C}_{\gamma},\|\cdot\|_{\gamma}\right)$ - несепарабельное банахово пространство. Очевидно, что для $x \in \mathscr{C}_{\gamma}, 0 \leqslant \gamma \leqslant 1$, имеет место неравенство

$$
\|x\|_{\infty} \leqslant\|x\|_{\gamma}, \quad\|x\|_{\infty}:=\sup _{t \in[0,1]}|x(t)|
$$

Кроме того, легко видеть, что в случае $\gamma=0$ выполнены соотношения

$$
\|x\|_{\infty} \leqslant\|x\|_{0} \leqslant 2\|x\|_{\infty}
$$

Неравенства (1.4) и (1.5) устанавливают связь между оценками нормы супремума и нормы Гёльдера. Отметим, что для эквивалентных норм асимптотики одних и тех же распределений, как правило, отличаются. Укажем также, что точная асимптотика вероятности (1.1) для нормы Гёльдера с $\gamma=0$ (так называемый "размах процесса") ранее была исследована в [24] для гауссовского стационарного процесса.

Пусть $w(t)$ - стандартный винеровский процесс, $w_{0}(t)=w(t)-t w(1), t \in[0,1],-$ броуновский мост. Напомним, что траектории винеровского процесса и броуновского моста принадлежат пространству $\mathscr{C}_{\gamma}$ при любом $0 \leqslant \gamma<1 / 2$ (см., например, $[4, \S 4.4$, c. 81$])$.

TЕОРема 1.1. Пусть $0 \leqslant \gamma<1 / 2$. Тогда при $u \rightarrow \infty$ имеет место соотношение

$$
\mathrm{P}\left\{\|w\|_{\gamma}>u\right\}=\exp \left\{-\frac{u^{2}}{2}\right\} \frac{8 \sqrt{2}}{\sqrt{\pi} u}\left(\frac{1-\gamma}{1-2 \gamma}\right)^{2}(1+o(1))
$$


Teорема 1.2. Пусть $0 \leqslant \gamma<1 / 2$. Тогда при $u \rightarrow \infty$ имеет место соотношение

$$
\mathrm{P}\left\{\left\|w_{0}\right\|_{\gamma}>u\right\}=\exp \left\{-\frac{u^{2}}{2 \tau^{2}}\right\} u^{2} \sqrt{2} \frac{(2-2 \gamma)^{7 / 2-2 \gamma}}{(1-2 \gamma)^{5 / 2-2 \gamma}}(1+o(1))
$$

гдe

$$
\tau^{2}=\frac{(1-2 \gamma)^{1-2 \gamma}}{(2-2 \gamma)^{2-2 \gamma}}
$$

Рассмотрим задачу вычисления асимптотики вероятности (1.1) для следуюшего важного класса гауссовских процессов.

Дробным броуновским движением (ДБД) с показателем $0<\alpha \leqslant 2$ называется гауссовский процесс (со стационарными прирашениями) $w_{\alpha}(t), t \in \mathbb{R}$, имеющий нулевое среднее и ковариационную функцию

$$
\mathrm{E} w_{\alpha}(t) w_{\alpha}(s)=\frac{1}{2}\left(|t|^{\alpha}+|s|^{\alpha}-|t-s|^{\alpha}\right), \quad t, s \in \mathbb{R} .
$$

Очевидно, из (1.9) следует равенство

$$
\mathrm{E}\left(w_{\alpha}(t)-w_{\alpha}(s)\right)^{2}=|t-s|^{\alpha}, \quad t, s \in[0,1] .
$$

Отметим, что процесс $w_{1}(t)$ (при $\alpha=1$ ) представляет собой стандартный винеровский процесс $w(t)$, или броуновское движение. Также укажем на отличия в свойствах процессов ДБД при $1<\alpha \leqslant 2, \alpha=1$ и $0<\alpha<1$ (последний случай наиболее сложен для вычислений).

Известно, что траектории процесса $w_{\alpha}(t)$ принадлежат пространству $\mathscr{C}_{\gamma}$ при любом $0 \leqslant \gamma<\alpha / 2$. Это можно доказать, проверив с учетом равенства (1.10) выполнение достаточного условия гёльдеровости траекторий (см. [4, с. 81]).

Процессы ДБД по сушеству впервые рассмотрены в неявном виде в работе [14]. Они применяются во многих задачах теории вероятностей [15], [16], в частности в теории больших уклонений гауссовских процессов в норме супремума.

Пусть

$$
\chi_{\alpha}(t):=\sqrt{2} w_{\alpha}(t)-|t|^{\alpha}, \quad t \in \mathbb{R} .
$$

Основная константа теории экстремальных значений гауссовских процессов определяется следуюшим образом (см. [9], [10], [17], [18]):

$$
0<H_{\alpha}=\lim _{S \rightarrow \infty} \frac{1}{S} \mathrm{E} \exp \left\{\sup _{t \in[0, S]} \chi_{\alpha}(t)\right\}<\infty .
$$

Сушествование предела в (1.12) впервые было доказано Дж. Пикандсом в [17].

Известно, что $H_{1}=1$ и $H_{2}=\pi^{-1 / 2}$. Удобных формул для выгисления константы $H_{\alpha}$ при остальных значениях $\alpha$ не сушествует. Некоторые оценки получены в работе [19], где, в частности, доказано неравенство

$$
0.12 \leqslant H_{\alpha} \leqslant 3.1 \text { при } 1<\alpha<2
$$

(см. также [20]).

В настоящей работе докажем, что константы типа Пикандса определяют также и поведение вероятностей больших уклонений гауссовских процессов в гёльдеровской норме. 
Teopema 1.3. (i) Пусть $1<\alpha \leqslant 2 u 0 \leqslant \gamma<\alpha / 2$. Тогда при $u \rightarrow \infty$ имеет место соотношение

$$
\mathrm{P}\left\{\left\|w_{\alpha}\right\|_{\gamma}>u\right\}=\exp \left\{-\frac{u^{2}}{2}\right\} u^{-1} 2 \sqrt{2} \pi^{-1 / 2}(1+o(1)) .
$$

(ii) Пусть $0<\alpha<1 u 0 \leqslant \gamma<\alpha / 2$. Тогда при $u \rightarrow \infty$ выполнено соотношение

$$
\mathrm{P}\left\{\left\|w_{\alpha}\right\|_{\gamma}>u\right\}=\exp \left\{-\frac{u^{2}}{2}\right\} u^{4 / \alpha-5} 2^{7 / 2-2 / \alpha} \pi^{-1 / 2}(\alpha-2 \gamma)^{-2} H_{\alpha}^{2}(1+o(1)) .
$$

Интересно сравнить теорему 1.3 с соответствуюшим результатом, приведенньгм ниже, для процессов ДБД и нормы супремума.

Tеорема 1.4. (i) Пусть $1<\alpha \leqslant 2$. Тогда при $u \rightarrow \infty$ имеет место соотношение

$$
\begin{aligned}
\mathrm{P}\left\{\sup _{t \in[0,1]}\left|w_{\alpha}(t)\right|>u\right\} & =2 \mathrm{P}\left\{\sup _{t \in[0,1]} w_{\alpha}(t)>u\right\}(1+o(1)) \\
& =\exp \left\{-\frac{u^{2}}{2}\right\} u^{-1} \sqrt{2} \pi^{-1 / 2}(1+o(1)) .
\end{aligned}
$$

(ii) Пусть $0<\alpha<1$. Тогда при $u \rightarrow \infty$ выполнено соотношение

$$
\begin{aligned}
& \mathrm{P}\left\{\sup _{t \in[0,1]} \mid\right.\left.w_{\alpha}(t) \mid>u\right\}=2 \mathrm{P}\left\{\sup _{t \in[0,1]} w_{\alpha}(t)>u\right\}(1+o(1)) \\
&=\exp \left\{-\frac{u^{2}}{2}\right\} u^{2 / \alpha-3} 2^{1 / 2-1 / \alpha} \pi^{-1 / 2} \alpha^{-1} H_{\alpha}(1+o(1)) .
\end{aligned}
$$

Асимптотики (1.15) и (1.16) несложно получить на основе общей теоремы 8.1 из [9]. Результат теоремы 1.4 ранее не был известен. Как видим, асимптотики (1.13) и (1.15) от $\alpha$ и $\gamma$ не зависят.

Рассмотрим еще два родственных класса гауссовских стационарных процессов. Пусть $0<\alpha \leqslant 1$. Положим

$$
R_{\alpha}(t):=1-|t|^{\alpha}, \text { если }|t| \leqslant 1 .
$$

На основе теорем Пойа и Бохнера-Хинчина в работе [21] доказано, что следуюшие две функции являются ковариационными функциями, определенными на всей оси:

$$
\begin{aligned}
& R_{\alpha, 1}(t)= \begin{cases}R_{\alpha}(t), & \text { если }|t| \leqslant 1, \\
0, & \text { если }|t|>1 ;\end{cases} \\
& R_{\alpha, 2}(t)= \begin{cases}R_{\alpha}(t), & \text { если }|t| \leqslant 1, \\
\text { периодически продолжена, } & \text { если }|t|>1 .\end{cases}
\end{aligned}
$$

Обозначим через $\xi_{\alpha}(t)$ и $\eta_{\alpha}(t), t \in \mathbb{R}$, гауссовские стационарные процессы с нулевым средним значением и ковариационными функциями $R_{\alpha, 1}(t)$ и $R_{\alpha, 2}(t)$ соответственно. В силу (1.17)-(1.19) и теоремы из [4, с. 180] заключаем, что траектории гауссовских процессов $\xi_{\alpha}(t)$ и $\eta_{\alpha}(t), t \in[0,1]$, принадлежат гёльдеровскому пространству $\mathscr{C}_{\gamma}$ при любом $0 \leqslant \gamma<\alpha / 2$. 
TЕОРема 1.5. Пусть $\zeta_{\alpha}(t)$ обозначает один из процессов $\xi_{\alpha}(t)$ или $\eta_{\alpha}(t)$. Тогда для любого и $>0$ выполнено равенство

$$
\mathrm{P}\left\{\left\|\zeta_{\alpha}\right\|_{\gamma}>u\right\}=\mathrm{P}\left\{\left\|w_{\alpha}\right\|_{\gamma}>u / \sqrt{2}\right\}
$$

СлЕДСТвИЕ 1.1. Для прочесса $\zeta_{\alpha}(t)$ из теоремы 1.5 справедливы следующие утверждения.

(i) Пусть $0<\alpha<1 u 0 \leqslant \gamma<\alpha / 2$. Тогда при $u \rightarrow \infty$ имеет место соотношение

$$
\mathrm{P}\left\{\left\|\zeta_{\alpha}\right\|_{\gamma}>u\right\}=\exp \left\{-\frac{u^{2}}{4}\right\} u^{4 / \alpha-5} 2^{6-4 / \alpha} \pi^{-1 / 2}(\alpha-2 \gamma)^{-2} H_{\alpha}^{2}(1+o(1)) .
$$

(ii) Пусть $0 \leqslant \gamma<1 / 2$. Тогда при $u \rightarrow \infty$ выполнено соотношение

$$
\mathrm{P}\left\{\left\|\zeta_{1}\right\|_{\gamma}>u\right\}=\exp \left\{-\frac{u^{2}}{4}\right\} u^{-1} 16 \pi^{-1 / 2}\left(\frac{1-\gamma}{1-2 \gamma}\right)^{2}(1+o(1)) .
$$

Результаты о малых уклонениях процессов $w_{\alpha}, \xi_{\alpha}(t)$ и $\eta_{\alpha}(t)$ в различных нормах (в том числе и гёльдеровской) см. в [21], [22, §4]. Теоремы о больших уклонениях для гауссовских процессов и полей в $L^{p}$-нормах доказаны в [30]-[32].

Как будет видно из приводимых ниже доказательств, результаты типа теорем 1.1-1.5 могут быть получены и для других гауссовских процессов, корреляционная функция которых удовлетворяет так называемому условию локальной стационарности (см. формулу (2.2) ниже). Возможны также обобщения на гауссовские случайные поля.

\section{§2. Основные теоремы метода двойных сумм}

Изложим основные результаты из [6]-[8], необходимые для доказательств теорем 1.1-1.5.

Пусть $X(t), t \in T,-$ гауссовское поле с непрерывными траекториями и нулевым средним, заданное на компакте $T \subset \mathbb{R}^{k}$. Пусть $\sigma^{2}(t)$ - дисперсия поля $X(t)$, $R(t, s)=\mathrm{E} X(t) X(s)$ - его ковариационная функция, $r(t, s)=R(t, s) /(\sigma(t) \sigma(s))$ его коррелящионная функция. Пусть задан вектор $a=\left(\alpha_{1}, \ldots, \alpha_{k}\right)$ с положительными координатами. Для вектора $t=\left(t_{1}, \ldots, t_{k}\right)$ определим величину

$$
|t|_{a}:=\sum_{i=1}^{k}\left|t_{i}\right|^{\alpha_{i}} .
$$

Ниже $|t-s|$ обозначает евклидово расстояние между векторами $t, s \in T$.

Введем следующие условия.

(B1) Дисперсия $\sigma^{2}(t)$ непрерьвна на $T$ и достигает своего максимума $\sigma^{2}$ в единственной точке $t_{0}$, причем имеет место разложсение

$$
\sigma(t)=\sigma-\left|A\left(t-t_{0}\right)\right|_{\beta}(1+o(1)), \quad t \rightarrow t_{0},
$$


где $A$ - некоторая невырожденная $(k \times k)$-матрица, $\beta=\left(\beta_{1}, \ldots, \beta_{k}\right), \beta_{i}>0$, $i=1, \ldots, k$.

(В2) Условие локальной стационарности: имеет место соотношение

$$
r(t, s)=1-|D(t-s)|_{a}(1+o(1)), \quad|t-s| \rightarrow 0, \quad t, s \rightarrow t_{0},
$$

где вектор а определен выше, $D$ - некоторая невырожденная $(k \times k)$-матрица.

(В3) Условие регулярности: найдутся $L>0, \nu>0 u \varepsilon>0$ такие, ито для всех $t, s \in T,|t-s|<\varepsilon$, имеет место неравенство

$$
\mathrm{E}(X(t)-X(s))^{2} \leqslant L \sum_{i=1}^{k}\left|t_{i}-s_{i}\right|^{\nu}, \quad t=\left(t_{1}, \ldots, t_{k}\right), \quad s=\left(s_{1}, \ldots, s_{k}\right) .
$$

ЗАМЕЧАнИЕ 2.1. Из условия (В3) следует выборочная непрерывность гауссовского поля $X(t), t \in T$. Это доказано, например, в [25, с. 174, формула (10)].

Обозначим через $\chi(t), t \in \mathbb{R}^{k}$, гауссовское поле с п.н. непрерывными траекториями, для которого

$$
\mathrm{E} \chi(t)=-|t|_{a}, \quad \operatorname{Cov}(\chi(t), \chi(s))=|t|_{a}+|s|_{a}-|t-s|_{a} .
$$

Поле $\chi(t)$ назьвается многопараметрическим дробным броуновским движением (со сносом), или полем Леви-Шенберга. При $k=1$ и $\alpha_{1}=\alpha$ поле (2.4) совпадает с процессом $\chi_{\alpha}$ из (1.11).

В статье [7, теорема 1.2, п. (ii)] было доказано существование предела для невырожденной матрицы $C$ :

$$
0<H_{a}^{b}(C):=\lim _{S \rightarrow \infty} \mathrm{E} \exp \left\{\sup _{t \in[-S, S]^{k}}\left(\chi(C t)-\sum_{i=1}^{k} b_{i}\left|t_{i}\right|^{\alpha_{i}}\right)\right\}<\infty
$$

где $b=\left(b_{1}, \ldots, b_{k}\right), b_{i}>0, i=1, \ldots, k$.

Аналогично доказывается существование предела

$0<\widetilde{H}_{a}^{b}(C):=\lim _{S \rightarrow \infty} \mathrm{E} \exp \left\{\sup _{t \in[0, S]^{k}}\left(\chi(C t)-\sum_{i=1}^{k} b_{i}\left|t_{i}\right|^{\alpha_{i}}\right)\right\}<\infty, \quad b=\left(b_{1}, \ldots, b_{k}\right)$

(случай $k=1$ подробно рассмотрен в [9, теорема 8.1]).

Несложно показать, что для диагональной матрищы $C=\operatorname{diag}\left(c_{1}, \ldots, c_{k}\right), c_{i}>0$, $i=1, \ldots, k$, справедливо равенство

$$
\widetilde{H}_{a}^{b}(C)=\prod_{i=1}^{k} \widetilde{H}_{\alpha_{i}}^{b_{i}}\left(c_{i}\right)
$$


Tеорема 2.1. Пусть $T=[0,1]^{k} u$ для поля $X(t), t \in T$, выполнены сформулированные выше условия, в том числе условия (B1)-(B3). Пусть матрицы $A$ и $D$ являются диагональными $, A=\operatorname{diag}\left(a_{1}, \ldots, a_{k}\right), D=\operatorname{diag}\left(d_{1}, \ldots, d_{k}\right)$, $a_{i}>0, d_{i}>0, i=1, \ldots, k$. Предположим, что единственная точка максимума дисперсии $t_{0}$ является одной из вершин куба $[0,1]^{k}$.

Тогда при и $\rightarrow \infty$ справедливы следующие утверждения.

(i) Пусть $\alpha_{i}<\beta_{i}, i=1, \ldots, k$. Тогда имеет место соотношение

$$
\begin{aligned}
\mathrm{P}\left\{\sup _{t \in T}|X(t)|>u\right\}=2 \mathrm{P}\left\{\sup _{t \in T} X(t)>u\right\}(1+o(1)) \\
=\exp \left\{-\frac{1}{2} \sigma^{-2} u^{2}\right\} u^{-1} \sqrt{2} \sigma \pi^{-1 / 2} \\
\quad \times \prod_{i=1}^{k}\left[\left(\frac{u}{\sigma}\right)^{2 / \alpha_{i}}\left(\frac{u^{2}}{\sigma^{3}}\right)^{-1 / \beta_{i}} \frac{d_{i}}{\beta_{i} a_{i}} \Gamma\left(\frac{1}{\beta_{i}}\right) H_{\alpha_{i}}\right](1+o(1)) .
\end{aligned}
$$

(ii) Пусть $\alpha_{i}=\beta_{i}, i=1, \ldots, k$. Тогда

$$
\begin{aligned}
\mathrm{P}\left\{\sup _{t \in T}|X(t)|>u\right\}=2 \mathrm{P}\left\{\sup _{t \in T} X(t)>u\right\}(1+o(1)) \\
=\exp \left\{-\frac{1}{2} \sigma^{-2} u^{2}\right\} u^{-1} \sqrt{2} \sigma \pi^{-1 / 2} \prod_{i=1}^{k} \widetilde{H}_{\alpha_{i}}^{1 / \sigma}\left(\frac{d_{i}}{a_{i}}\right)(1+o(1)) .
\end{aligned}
$$

(iii) Пусть $\alpha_{i}>\beta_{i}, i=1, \ldots, k$. Тогда выполнено соотношение

$$
\begin{array}{r}
\mathrm{P}\left\{\sup _{t \in T}|X(t)|>u\right\}=2 \mathrm{P}\left\{\sup _{t \in T} X(t)>u\right\}(1+o(1)) \\
=\exp \left\{-\frac{1}{2} \sigma^{-2} u^{2}\right\} u^{-1} \sqrt{2} \sigma \pi^{-1 / 2}(1+o(1)) .
\end{array}
$$

ЗАмЕЧАниЕ 2.2. Пусть в условиях любого из пунктов (i)-(iii) теоремы 2.1 дисперсия гауссовского поля $X(t)$ достигает своего абсолютного максимума в нескольких изолированных граничных точках, причем взаимные коррелящии значений поля в этих точках меньше единицы. Тогда нетрудно показать, что асимптотика вероятности (1.2) равна главному члену суммы асимптотик, вычисленных по соответствующим формулам теоремы 2.1 .

Доказательство утверждений типа теоремы 2.1 см. в [7, теорема 1.2], где подробно рассмотрен случай, когда точка $t_{0}$ является внутренней точкой множества $T$. При $k=1$ теорема 2.1 доказана в [9, теорема 8.1].

Первые равенства в формулах (2.6)-(2.8) имеют место в силу следующего утверждения.

ЛЕмма 2.1. В условиях теоремы 2.1 при $и \rightarrow \infty$ справедливо соотношение

$$
\mathrm{P}\left\{\sup _{t \in T}|X(t)|>u\right\}=2 \mathrm{P}\left\{\sup _{t \in T} X(t)>u\right\}(1+o(1)) .
$$


ЗАмЕчАниЕ 2.3. Если в условиях теоремы 2.1 для некоторого $\delta_{1}>0$ выполнено неравенство

$$
\mathrm{E} X(t) X(s) /[\sigma(t) \sigma(s)]>-1+\delta_{1} \quad \text { для всех } t, s \in T,
$$

то справедливость формулы (2.9) следует из [9, с. 64, следствие 6.4]. В ситуации, которая рассматривается в настоящей статье, условие (2.10) не выполняется.

$$
\begin{aligned}
& \text { ДокаЗАТЕЛЬСтво ЛЕммы 2.1. Имеем тождество } \\
& \mathrm{P}\left\{\sup _{t \in T}|X(t)|>u\right\}=\mathrm{P}\left\{\sup _{t \in T} X(t)>u\right\}+\mathrm{P}\left\{\sup _{s \in T}[-X(s)]>u\right\}-P_{0},
\end{aligned}
$$

где

$$
P_{0}:=\mathrm{P}\left\{\sup _{t \in T} X(t)>u, \sup _{s \in T}[-X(s)]>u\right\} .
$$

Обозначим через $Q(u)$ точную асимптотику вероятности $\mathrm{P}\left\{\sup _{t \in T} X(t)>u\right\}$. Покажем, что $P_{0}=o(Q(u))$ при $u \rightarrow \infty$. Для этого нам придется повторить некоторые оценки и выкладки работы [7].

Пусть функция $\delta_{0}(u)$ такова, что при $u \rightarrow \infty$ выполнены соотношения

$$
\delta_{0}(u) \downarrow 0, \quad u \delta_{0} \rightarrow 0, \quad u^{2} \delta_{0}(u) / \ln u \rightarrow \infty .
$$

Обозначим $T_{\delta_{0}}=\left\{t \in T:\left|A\left(t-t_{0}\right)\right|_{\beta} \leqslant \delta_{0}(u)\right\}$. Имеем

$$
\begin{aligned}
P_{0} \leqslant \mathrm{P}\left\{\sup _{t \in T_{\delta_{0}}} X(t)>u, \sup _{s \in T}[-X(s)]>u\right\} \\
+\mathrm{P}\left\{\sup _{t \in T \backslash T_{\delta_{0}}} X(t)>u, \sup _{s \in T}[-X(s)]>u\right\} .
\end{aligned}
$$

Введем гауссовское поле $Z(t, s):=X(t)-X(s)$ с непрерывными траекториями и нулевым средним, заданное на компакте $T \times T \subset \mathbb{R}^{2 k}$. Вторая вероятность в $(2.13)$ оценивается сверху величиной

$$
\mathrm{P}\left\{\sup _{(t, s) \in\left[T \backslash T_{\delta_{0}}\right] \times T} Z(t, s)>2 u\right\} .
$$

Подобно доказательству леммы 3.2 из [7], с использованием леммы 6.3 из [9], показывается, что вероятность (2.14) есть величина $o(Q(u))$.

Оценивая далее сверху первую вероятность в (2.13) и рассуждая аналогично, приходим к соотношению

$$
P_{0}=\mathrm{P}\left\{\sup _{t \in T_{\delta_{0}}} X(t)>u, \sup _{s \in T_{\delta_{0}}}[-X(s)]>u\right\}+o(Q(u))
$$

Оценка вероятности справа в (2.15) осушествляется по той же схеме, что и оценка двойной суммы в формуле (3.6) работы [7], с использованием леммы 6.6 из [9]. Таким образом, убеждаемся, что $P_{0}=o(Q(u))$ при $u \rightarrow \infty$.

Теперь формула (2.9) следует из (2.11), поскольку гауссовские поля $\{X(t)$, $t \in T\}$ и $\{-X(t), t \in T\}$ имеют одинаковые распределения. Лемма доказана.

Ниже, на основе теоремы 2.1 докажем теоремы 1.1, 1.3-1.5. Для доказательства теоремы 1.2 нам понадобится иной результат, в котором рассмотрен случай, когда дисперсия гауссовского поля достигает своего максимума на многообразии размерности $k-1$.

Сформулируем соответствуюшие условия. 
(C1) Дисперсия $\sigma^{2}(t)$ гауссовского непрерьвного поля $X(t), t \in T \subset \mathbb{R}^{k}$, достигает своего максимума $\sigma^{2}$ на множестве $T_{0}:=\{t \in T: G(t)=0\}$, причем для некоторого $\beta>0$ имеет место соотношение

$$
\sigma(t)=\sigma-|G(t)|^{\beta}(1+o(1)), \quad G(t) \rightarrow 0 .
$$

Пусть функция $G(t)$ удовлетворяет условиям теоремы о неявной функции:

а) для некоторого $\varepsilon>0$ функция $G(t)$ определена и непрерывна на множестве

$$
T_{0}^{\varepsilon}:=\left\{t \in T: \inf _{s \in T_{0}}|t-s| \leqslant \varepsilon\right\}
$$

b) имеет место представление $T_{0}^{\varepsilon}=\bigcup_{i=1}^{k} T_{i}$, причем для каждого $i$ существует и непрерьвна производная $\frac{\partial}{\partial t_{i}} G(t), t \in T_{i}$;

с) выполнено соотношение

$$
\frac{\partial}{\partial t_{i}} G(t) \neq 0, \quad t \in T_{i} \cap T_{0}, \quad i=1, \ldots, k
$$

(С2) Условие локальной стационарности: найдется такое $\varepsilon_{0}>0$, ито для всех $t, s \in T_{0}^{\varepsilon_{0}}$ выполнено соотношение

$$
r(t, s)=1-|D(t, s)(t-s)|_{a}(1+o(1)), \quad|t-s| \rightarrow 0,
$$

где вектор а определен выше, $D(t, s)=\operatorname{diag}\left(d_{1}(t, s), \ldots, d_{k}(t, s)\right)$ - диагональная матрица, әлементы $d_{i}(t, s)$ которой заданы и непрерывны на $T_{0}^{\varepsilon_{0}} \times T_{0}^{\varepsilon_{0}}$, причем

$$
\prod_{i=1}^{k} d_{i}(t, t) \neq 0, \quad t \in T_{0}^{\varepsilon_{0}} .
$$

(С3) Для некоторого открытого в $\mathbb{R}^{k}$ множества $U$ имеет место включение $T_{0} \subset \bar{U} \subset T$ (здесь $\bar{U}$ обозначает замикание множества $U$ ).

(C4) Найдется такое $\varepsilon_{1}>0$, что для любого $\kappa>0$ справедливо неравенство

$$
\sup \left\{R(t, s): t, s \in T_{0}^{\varepsilon_{1}},|t-s| \geqslant \kappa\right\}<\sigma^{2},
$$

где, как и ранее, $R(t, s)$ - ковариационная функиия поля $X(t)$.

Заметим, что при необходимости условие (C4) может быть ослаблено.

Tеорема 2.2. Пусть $X(t)$ - гауссовское поле с непрерывными траекториями и нулевым средним, заданное на компакте $T \subset \mathbb{R}^{k}$. Предположим, ито выполнены условия (C1)-(C4), (B3) и $\beta>\max _{1 \leqslant i \leqslant k} \alpha_{i}$. Пусть функиия $\delta(u)$ такова, что при $и \rightarrow \infty$ имеют место соотношения

$$
\delta(u) \downarrow 0, \quad u \delta^{\beta}(u) \rightarrow 0, \quad u^{2} \delta^{\beta}(u) / \ln u \rightarrow \infty .
$$


Тогда при $и \rightarrow \infty$ справедливо соотношение

$$
\begin{aligned}
\mathrm{P}\left\{\sup _{t \in T}|X(t)|>u\right\}=2 \mathrm{P}\left\{\sup _{t \in T} X(t)>u\right\}(1+o(1)) \\
=\exp \left\{-\frac{1}{2} \sigma^{-2} u^{2}\right\} u^{-1} \sqrt{2} \sigma \pi^{-1 / 2} \prod_{i=1}^{k}\left[\left(\frac{u}{\sigma}\right)^{2 / \alpha_{i}} H_{\alpha_{i}}\right] \\
\times \int_{T_{\delta}} \exp \left\{-u^{2} \sigma^{-3}|G(t)|^{\beta}\right\} \prod_{i=1}^{k}\left|d_{i}(t, t)\right| d t(1+o(1)),
\end{aligned}
$$

где $T_{\delta}=\{t \in T:|G(t)| \leqslant \delta(u)\}$.

Доказательство теоремы 2.2 содержится в работах $[7$, теорема 1.1$]$ и $[8$, теорема 2.1], где даны также ее применения к винеровскому полю. (Заметим, что в правую часть формулы (2.5) из [8] нужно дописать пропущенный множитель $1 /(n-1)$ !; см. также [23].)

\section{§3. Доказательства теорем 1.1, 1.3-1.5}

Докажем сначала теоремы 1.1 и 1.3.

Пусть $0<\alpha \leqslant 2$ и $0 \leqslant \gamma<\alpha / 2$. Доказательства утверждений теорем типа теорем 1.1-1.5 основаны на следуюшем приеме, использованном впервые в контексте гауссовских процессов в работе [24]. Введем гауссовское случайное поле $Y_{\alpha}(t)$ (зависимость от $\gamma$ не указываем), $t=\left(t_{1}, t_{2}\right)$, заданное на единичном квадрате $T=[0,1]^{2}$, следуюшим образом:

$$
Y_{\alpha}(t):= \begin{cases}\frac{w_{\alpha}\left(t_{1}\right)-w_{\alpha}\left(t_{2}\right)}{\left|t_{1}-t_{2}\right|^{\gamma}}, & \text { если } t \in[0,1]^{2}, t_{1} \neq t_{2}, \\ 0, & \text { если } t \in[0,1]^{2}, t_{1}=t_{2} .\end{cases}
$$

Поле $Y_{\alpha}(t)$ имеет нулевое среднее значение. Пусть точки $t=\left(t_{1}, t_{2}\right), s=\left(s_{1}, s_{2}\right)$ принадлежат квадрату $[0,1]^{2}$; тогда с учетом (1.9) легко вычислить, что ковариационная функция поля $Y_{\alpha}(t)$ имеет вид

$$
\mathrm{E} Y_{\alpha}(t) Y_{\alpha}(s)=\left\{\begin{array}{cl}
\frac{1}{2}\left|t_{2}-t_{1}\right|^{-\gamma}\left|s_{2}-s_{1}\right|^{-\gamma} & \\
\times\left[\left|t_{2}-s_{1}\right|^{\alpha}+\left|t_{1}-s_{2}\right|^{\alpha}\right. & \\
\left.\quad-\left|t_{1}-s_{1}\right|^{\alpha}-\left|t_{2}-s_{2}\right|^{\alpha}\right], & \text { если } t_{1} \neq t_{2}, s_{1} \neq s_{2}, \\
0 & \text { иначе. }
\end{array}\right.
$$

Непрерьвность траекторий поля $Y_{\alpha}(t)$ следует из доказанной ниже леммы 3.1 и замечания 2.1 .

Легко видеть, что для любого $u>0$ выполнено равенство

$$
\mathrm{P}\left\{\left\|w_{\alpha}\right\|_{\gamma}>u\right\}=\mathrm{P}\left\{\sup _{t \in T}\left|Y_{\alpha}(t)\right|>u\right\} .
$$

Применим к полю $Y_{\alpha}(t), t \in[0,1]^{2}$, теорему 2.1 вместе с замечанием 2.2. 
Дисперсия $\sigma_{\alpha}^{2}(t)$ поля $Y_{\alpha}(t)$ имеет вид $\sigma_{\alpha}^{2}(t)=\left|t_{1}-t_{2}\right|^{\alpha-2 \gamma}$ и достигает на множестве $[0,1]^{2}$ своего максимума $\sigma^{2}=1$ в двух точках: $t_{0}=(0,1)$ и $\tilde{t}_{0}=(1,0)$. Ограничимся рассмотрением только точки $t_{0}$, поскольку вычисления для точки $\tilde{t}_{0}$ (симметричной точке $t_{0}$ относительно главной диагонали единичного квадрата) те же самые.

Используя формулу Тейлора, несложно получить разложение

$$
\sigma_{\alpha}(t)=1-|A t|_{\mathbf{l}}(1+o(1)), \quad t \rightarrow t_{0},
$$

где $\mathbf{l}=(1,1), A=2^{-1}(\alpha-2 \gamma) I, I-$ единичная матрица. Так же просто записать требуемое разложение для корреляционной функции $r_{\alpha}(t, s)$ поля $Y_{\alpha}(t)$ :

$$
r_{\alpha}(t, s)=1-|D(t-s)|_{a}(1+o(1)), \quad|t-s| \rightarrow 0, \quad t, s \rightarrow t_{0},
$$

где $a=(\alpha, \alpha), D=(1 / 2)^{1 / \alpha} I$.

Докажем выполнение неравенства из условия (В3).

Лемма 3.1. Найдется $\varepsilon>0$ такое, что для всех $t=\left(t_{1}, t_{2}\right), s=\left(s_{1}, s_{2}\right)$ uз $[0,1]^{2},|t-s|<\varepsilon$, имеет место неравенство

$$
\mathrm{E}\left(Y_{\alpha}(t)-Y_{\alpha}(s)\right)^{2} \leqslant 4 \sum_{i=1}^{2}\left|t_{i}-s_{i}\right|^{\alpha-2 \gamma} .
$$

ДокАЗАТЕЛЬСТво. Без ограничения общности предположим, что

$$
s_{1}<t_{1}<s_{2}<t_{2}, \quad t_{2}-t_{1}<s_{2}-s_{1} .
$$

Имеем

$$
\begin{aligned}
\mathrm{E}\left(Y_{\alpha}(t)-Y_{\alpha}(s)\right)^{2}= & {\left[\left(t_{2}-t_{1}\right)^{\alpha-2 \gamma}-\frac{\left(t_{2}-s_{1}\right)^{\alpha}}{\left(t_{2}-t_{1}\right)^{\gamma}\left(s_{2}-s_{1}\right)^{\gamma}}\right] } \\
& +\left[\left(s_{2}-s_{1}\right)^{\alpha-2 \gamma}-\frac{\left(s_{2}-t_{1}\right)^{\alpha}}{\left(t_{2}-t_{1}\right)^{\gamma}\left(s_{2}-s_{1}\right)^{\gamma}}\right] \\
& +\left[\frac{\left(t_{1}-s_{1}\right)^{\alpha}}{\left(t_{2}-t_{1}\right)^{\gamma}\left(s_{2}-s_{1}\right)^{\gamma}}\right]+\left[\frac{\left(t_{2}-s_{2}\right)^{\alpha}}{\left(t_{2}-t_{1}\right)^{\gamma}\left(s_{2}-s_{1}\right)^{\gamma}}\right] \\
\equiv & J_{1}+J_{2}+J_{3}+J_{4} .
\end{aligned}
$$

Оценим сверху каждое из четырех слагаемых в правой части (3.5). Имеем согласно формуле (3.4)

$$
\begin{aligned}
J_{1}= & \left(t_{2}-t_{1}\right)^{-2 \gamma}\left(s_{2}-s_{1}\right)^{-\gamma}\left\{\left(t_{2}-t_{1}\right)^{\alpha}\left[\left(s_{2}-s_{1}\right)^{\gamma}-\left(t_{2}-t_{1}\right)^{\gamma}\right]\right. \\
& \left.+\left(t_{2}-t_{1}\right)^{\gamma}\left[\left(t_{2}-t_{1}\right)^{\alpha}-\left(t_{2}-s_{1}\right)^{\alpha}\right]\right\} \\
\leqslant & \left(t_{2}-t_{1}\right)^{\alpha-2 \gamma}\left(s_{2}-s_{1}\right)^{-\gamma}\left[\left(s_{2}-s_{1}\right)^{\gamma}-\left(t_{2}-t_{1}\right)^{\gamma}\right] .
\end{aligned}
$$

Используя дважды элементарное неравенство

$$
|x+y|^{\gamma} \leqslant|x|^{\gamma}+|y|^{\gamma}, \quad x, y \in \mathbb{R}, \quad 0 \leqslant \gamma<1,
$$


получим

$$
\left(s_{2}-s_{1}\right)^{\gamma}-\left(t_{2}-t_{1}\right)^{\gamma} \leqslant\left|\left(s_{2}-s_{1}\right)-\left(t_{2}-t_{1}\right)\right|^{\gamma} \leqslant\left(t_{2}-s_{2}\right)^{\gamma}+\left(t_{1}-s_{1}\right)^{\gamma} \text {. }
$$

Оценивая последнее выражение в (3.6) посредством (3.7), находим

$$
J_{1} \leqslant\left(t_{2}-t_{1}\right)^{\alpha-2 \gamma}\left(s_{2}-s_{1}\right)^{-\gamma}\left[\left(t_{1}-s_{1}\right)^{\gamma}+\left(t_{2}-s_{2}\right)^{\gamma}\right] \text {. }
$$

Учитывая неравенства (3.4), для первого слагаемого справа в (3.8) имеем оценку

$$
\begin{aligned}
\left(t_{2}-t_{1}\right)^{\alpha-2 \gamma}\left(s_{2}-s_{1}\right)^{-\gamma}\left(t_{1}-s_{1}\right)^{\gamma} & =\frac{\left(t_{2}-t_{1}\right)^{\alpha-2 \gamma}}{\left(s_{2}-s_{1}\right)^{\alpha-2 \gamma}} \frac{\left(t_{1}-s_{1}\right)^{3 \gamma-\alpha}}{\left(s_{2}-s_{1}\right)^{3 \gamma-\alpha}}\left(t_{1}-s_{1}\right)^{\alpha-2 \gamma} \\
& \leqslant\left(t_{1}-s_{1}\right)^{\alpha-2 \gamma}
\end{aligned}
$$

В силу (3.4) аналогичная оценка верна и для второго слагаемого в (3.8):

$$
\begin{aligned}
\left(t_{2}-t_{1}\right)^{\alpha-2 \gamma}\left(s_{2}-s_{1}\right)^{-\gamma}\left(t_{2}-s_{2}\right)^{\gamma} & =\frac{\left(t_{2}-t_{1}\right)^{\alpha-2 \gamma}}{\left(s_{2}-s_{1}\right)^{\alpha-2 \gamma}} \frac{\left(t_{2}-s_{2}\right)^{3 \gamma-\alpha}}{\left(s_{2}-s_{1}\right)^{3 \gamma-\alpha}}\left(t_{2}-s_{2}\right)^{\alpha-2 \gamma} \\
& \leqslant\left(t_{2}-s_{2}\right)^{\alpha-2 \gamma}
\end{aligned}
$$

Из (3.8)-(3.10) получаем

$$
J_{1} \leqslant\left(t_{1}-s_{1}\right)^{\alpha-2 \gamma}+\left(t_{2}-s_{2}\right)^{\alpha-2 \gamma} .
$$

Аналогично выводятся остальные оценки (рассуждения для $J_{3}, J_{4}$ совсем простые):

$$
J_{k} \leqslant\left(t_{1}-s_{1}\right)^{\alpha-2 \gamma}+\left(t_{2}-s_{2}\right)^{\alpha-2 \gamma}, \quad k=2,3,4 .
$$

Из соотношений $(3.5),(3.11),(3.12)$ получаем неравенство (3.3).

Лемма 3.1 , а вместе с ней и выборочная непрерывность поля $Y_{\alpha}(t)$ доказаны.

Легко найти, что взаимная корреляция значений поля $Y_{\alpha}(t)$ в точках $t_{0}$ и $\tilde{t}_{0}$ равна -1 :

$$
\mathrm{E} Y_{\alpha}\left(t_{0}\right) Y_{\alpha}\left(\tilde{t}_{0}\right) /\left[\sigma_{\alpha}\left(t_{0}\right) \sigma_{\alpha}\left(\tilde{t}_{0}\right)\right]=-1
$$

Таким образом, заключение замечания 2.2 справедливо для поля $Y_{\alpha}(t), t \in[0,1]^{2}$, но, как видим, условие (2.10) не выполнено.

Далее рассмотрим последовательно три случая.

ДОКАЗАТЕЛЬСТВО ТЕОРЕМЫ 1.1. Пусть $\alpha=1$. Из разложений $(3.1)$ и $(3.2)$ и леммы 3.1 следует, что для точки $t_{0}=(0,1)$ выполнены условия п. (ii) теоремы 2.1 с показателями $\alpha_{i}=\beta_{i}=1, i=1,2$, и матрицами $A=2^{-1}(1-2 \gamma) I, D=2^{-1} I$. То же самое заключение с теми же величинами верно и для точки $\tilde{t}_{0}=(1,0)$.

Согласно определению поля $Y_{1}(t)$, формуле $(2.7)$ и замечанию 2.2 при $u \rightarrow \infty$ имеем

$$
\begin{aligned}
\mathrm{P}\left\{\|w\|_{\gamma}>u\right\} & =\mathrm{P}\left\{\sup _{t \in T}\left|Y_{1}(t)\right|>u\right\}=2 \mathrm{P}\left\{\sup _{t \in T} Y_{1}(t)>u\right\}(1+o(1)) \\
& =\exp \left\{-\frac{u^{2}}{2}\right\} u^{-1} \frac{2 \sqrt{2}^{-1 / 2}}{\pi}\left[\widetilde{H}_{1}^{1}\left(\frac{1}{1-2 \gamma}\right)\right]^{2}(1+o(1)) .
\end{aligned}
$$


Осталось вычислить константу $\widetilde{H}_{1}^{1}(1 /(1-2 \gamma))$ в формуле (3.13). Это сделано в следующей ниже лемме.

Обозначим $\widetilde{H}_{1}^{b}:=\widetilde{H}_{1}^{b}(1), b>0$. Тогда, очевидно,

$$
\widetilde{H}_{1}^{b}(c)=\widetilde{H}_{1}^{b / c}, \quad b>0, \quad c>0 .
$$

Следовательно,

$$
\widetilde{H}_{1}^{1}(1 /(1-2 \gamma))=\widetilde{H}_{1}^{1-2 \gamma} .
$$

Лемма 3.2. Пусть $b>0$. Тогда

$$
\widetilde{H}_{1}^{b}=\frac{b+1}{b} .
$$

ДоКАЗАТЕльСТво. Используя формулы $(1.11),(2.5)$ и свойство автомодельности винеровского процесса $(\sqrt{2} w(t) \stackrel{(\mathrm{d})}{=} w(2 t))$, имеем

$$
\widetilde{H}_{1}^{b}=\lim _{S \rightarrow \infty} \mathrm{E} \exp \left\{\sup _{t \in[0,2 S]}(w(t)-t(b+1) / 2)\right\} .
$$

Как известно, при $\mu<0$ справедлива следующая формула (см., например, [26, с. 252, пример № 1.1.4(1)]):

$$
\mathrm{P}\left\{\sup _{t \in[0, \infty)}(w(t)+\mu t) \geqslant y\right\}=\exp \{2 \mu y\}, \quad y \geqslant 0 .
$$

Положив в $(3.17) \mu=-(b+1) / 2$, на основе теоремы Лебега о мажорированной сходимости из соотношений $(3.16),(3.17)$ получаем

$$
\widetilde{H}_{1}^{b}=\mathrm{E} \exp \left\{\sup _{t \in[0, \infty)}(w(t)-t(b+1) / 2)\right\}=\frac{b+1}{b} .
$$

Лемма доказана.

Соотношения (3.13)-(3.15) при $b=1-2 \gamma$ завершают доказательство теоремы 1.1.

ДОКАЗАТЕЛЬСТВО ТЕОРЕМЫ 1.3 проводим аналогично доказательству теоремы 1.1, используя формулы (3.1), (3.2), лемму 3.1 и замечание 2.2. Утверждение п. (i) получаем на основе п. (iii) теоремы 2.1, а утверждение п. (ii) - на основе п. (i) теоремы 2.1.

ДОКАЗАТЕЛЬСТВО ТЕОРЕМЫ 1.4. Применим теорему 2.1 при $k=1$. Дисперсия $\theta_{\alpha}^{2}(t)=t^{\alpha}$ процесса ДБД $w_{\alpha}(t)$ достигает на $[0,1]$ своего максимума $\theta^{2}=1$ в единственной граничной точке $t_{0}=1$. Имеем разложение для корня из дисперсии:

$$
\theta_{\alpha}(t)=1-\frac{\alpha}{2}|t-1|(1+o(1)), \quad t \rightarrow 1 .
$$

Нужное разложение для корреляционной функции $\rho_{\alpha}(t, s)$ процесса $w_{\alpha}(t)$ имеет вид

$$
\rho_{\alpha}(t, s)=1-\frac{1}{2}|t-s|^{\alpha}(1+o(1)), \quad|t-s| \rightarrow 0, \quad t, s \rightarrow 1 .
$$

Неравенство из условия (В3) вьполнено в силу соотношения (1.10).

Используя соотношения (3.18), (3.19), применим теорему 2.1. Формулу (1.15) получаем с помошью утверждения (iii) этой теоремы, формулу (1.16) - на основе утверждения (i). Теорема доказана. 
ДОКАЗАТЕЛЬСТВО ТЕОРЕМЫ 1.5 . Пусть $0<\alpha \leqslant 1$ и $0 \leqslant \gamma<\alpha / 2$. Введем гауссовское случайное поле $Z_{\alpha}(t)$ (зависимость от $\gamma$ опять не указываем), $t=\left(t_{1}, t_{2}\right)$, заданное на единичном квадрате $[0,1]^{2}$ формулой

$$
Z_{\alpha}(t):= \begin{cases}\frac{\zeta_{\alpha}\left(t_{1}\right)-\zeta_{\alpha}\left(t_{2}\right)}{\left|t_{1}-t_{2}\right|^{\gamma}}, & \text { если } t \in[0,1]^{2}, t_{1} \neq t_{2}, \\ 0, & \text { если } t \in[0,1]^{2}, t_{1}=t_{2} .\end{cases}
$$

Поле $Z_{\alpha}(t)$ имеет нулевое среднее значение. Пусть $t=\left(t_{1}, t_{2}\right) \in[0,1]^{2}, s=$ $\left(s_{1}, s_{2}\right) \in[0,1]^{2} ;$ используя $(1.17)-(1.19)$, легко найти, что ковариационная функция поля $Z_{\alpha}(t)$ имеет вид

$$
\mathrm{E} Z_{\alpha}(t) Z_{\alpha}(s)=\left\{\begin{array}{cl}
\left|t_{2}-t_{1}\right|^{-\gamma}\left|s_{2}-s_{1}\right|^{-\gamma} & \\
\times\left[\left|t_{2}-s_{1}\right|^{\alpha}+\left|t_{1}-s_{2}\right|^{\alpha}\right. & \\
\left.\quad-\left|t_{1}-s_{1}\right|^{\alpha}-\left|t_{2}-s_{2}\right|^{\alpha}\right], & \text { если } t_{1} \neq t_{2}, s_{1} \neq s_{2}, \\
0 & \text { иначе. }
\end{array}\right.
$$

Сравнивая ковариационные функции полей $Z_{\alpha}(t)$ и $Y_{\alpha}(t)$, заключаем, что

$$
\mathrm{E} Z_{\alpha}(t) Z_{\alpha}(s)=2 \mathrm{E} Y_{\alpha}(t) Y_{\alpha}(s), \quad t, s \in[0,1]^{2} .
$$

Но тогда из леммы 3.1 следует непрерывность траекторий поля $Z_{\alpha}(t)$. Учитывая (3.21) и непрерывность центрированных гауссовских полей $Z_{\alpha}$ и $Y_{\alpha}$, приходим к выводу, что

$$
\left\{Z_{\alpha}(t), t \in[0,1]^{2}\right\} \stackrel{(\mathrm{d})}{=}\left\{\sqrt{2} Y_{\alpha}(t), t \in[0,1]^{2}\right\} .
$$

Из (3.20) и (3.22) для любого $u>0$ получаем

$$
\mathrm{P}\left\{\|\zeta\|_{\gamma}>u\right\}=\mathrm{P}\left\{\sup _{t \in T}\left|Z_{\alpha}(t)\right|>u\right\}=\mathrm{P}\left\{\sup _{t \in T}\left|Y_{\alpha}(t)\right|>u / \sqrt{2}\right\} .
$$

Отсюда следует формула (1.20). Теорема доказана.

Формулы (1.21), (1.22) выводятся на основе теорем 1.1, 1.3 (п. (ii)) и 1.5.

\section{§4. Доказательство теоремы 1.2}

Напомним, что броуновский мост $w_{0}(x), x \in[0,1],-$ это гауссовский процесс с нулевым средним значением и ковариационной функцией

$$
\mathrm{E} w_{0}(x) w_{0}(y)=\min (x, y)-x y, \quad x, y \in[0,1] .
$$

Теорему 1.2 будем доказывать по той же схеме, что и вьше.

Вновь введем гауссовское случайное поле $V(t)$ (зависимость от $\gamma$ не указываем), $t=\left(t_{1}, t_{2}\right)$, заданное на единичном квадрате $T=[0,1]^{2}$ формулой

$$
V(t):= \begin{cases}\frac{w_{0}\left(t_{1}\right)-w_{0}\left(t_{2}\right)}{\left|t_{1}-t_{2}\right|^{\gamma}}, & \text { если } t \in[0,1]^{2}, t_{1} \neq t_{2}, \\ 0, & \text { если } t \in[0,1]^{2}, t_{1}=t_{2} .\end{cases}
$$


Поле $V(t)$ имеет нулевое среднее значение. Пусть $t=\left(t_{1}, t_{2}\right) \in[0,1]^{2}, s=$ $\left(s_{1}, s_{2}\right) \in[0,1]^{2}$; используя (4.1), легко найти, что ковариационная функция поля $V(t)$ имеет вид

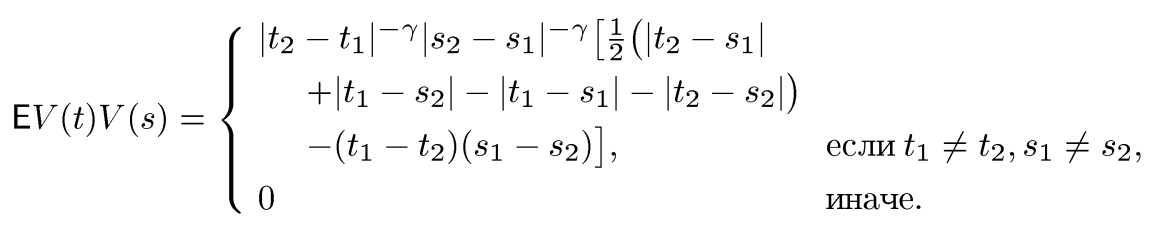

Сравнивая ковариационные функции полей $V(t)$ и $Y_{1}(t)$, заключаем, что

$$
\begin{gathered}
\mathrm{E} V(t) V(s)=\mathrm{E} Y_{1}(t) Y_{1}(s)-\left|t_{2}-t_{1}\right|^{-\gamma}\left|s_{2}-s_{1}\right|^{-\gamma}\left(t_{1}-t_{2}\right)\left(s_{1}-s_{2}\right), \\
t, s \in[0,1]^{2} .
\end{gathered}
$$

Кроме того,

$$
\mathrm{E} V^{2}(t)=\mathrm{E} Y_{1}^{2}(t)-\left|t_{1}-t_{2}\right|^{2-2 \gamma}, \quad t \in[0,1]^{2} .
$$

Из (4.4) и (4.5) получаем равенство

$$
\begin{gathered}
\mathrm{E}(V(t)-V(s))^{2}=\mathrm{E}\left(Y_{1}(t)-Y_{1}(s)\right)^{2}-\left[\left|t_{1}-t_{2}\right|^{1-\gamma}-\left|s_{1}-s_{2}\right|^{1-\gamma}\right]^{2}, \\
t, s \in[0,1]^{2} .
\end{gathered}
$$

Отсюда и из леммы 3.1 следует непрерывность траекторий поля $V(t)$ и вьполнение для него условия (B3).

Далее, вновь используем очевидное равенство, верное для любого $u>0$ :

$$
\mathrm{P}\left\{\left\|w_{0}\right\|_{\gamma}>u\right\}=\mathrm{P}\left\{\sup _{t \in T}|V(t)|>u\right\} .
$$

Обозначим

$$
1 \geqslant \lambda:=1-2 \gamma>0 .
$$

Лемма 4.1. Дисперсия $\tau^{2}(t)$ поля $V(t)$ имеет вид

$$
\tau^{2}(t)=\left|t_{1}-t_{2}\right|^{\lambda}-\left|t_{1}-t_{2}\right|^{1+\lambda}
$$

и достигает на множестве $[0,1]^{2}$ своего максимума

$$
\tau^{2}=\lambda^{\lambda} /(\lambda+1)^{\lambda+1}
$$

на одномерном многообразии

$$
T_{0}=\left\{t \in[0,1]^{2}:\left|t_{1}-t_{2}\right|=\lambda /(\lambda+1)\right\} .
$$

ДокаЗАТЕЛЬСТво. Обозначим $\left|t_{1}-t_{2}\right|=x \in[0,1]$. Очевидно, максимум функции (4.9) совпадает с максимумом функции $x^{\lambda}-x^{1+\lambda}, x \in[0,1]$. Но последний максимум достигается в единственной точке $x_{0}=\lambda /(\lambda+1)$. Отсюда следует утверждение леммы. 
Воспользуемся теперь теоремой 2.2. В обозначениях этой теоремы положим $G(t)=\left|t_{1}-t_{2}\right|-\lambda /(\lambda+1)$.

Несложно записать требуемое разложение из условия $(\mathrm{C} 1)$ для корня из дисперсии:

$$
\tau(t)=\tau-|\sqrt{K} G(t)|^{2}(1+o(1)), \quad G(t) \rightarrow 0,
$$

где

$$
K=\frac{1}{4} \lambda^{(\lambda-2) / 2}(\lambda+1)^{(3-\lambda) / 2} .
$$

Разложение из условия (C2) для коррелящионной функции $r_{0}(t, s)$ поля $V(t)$ имеет вид

$$
r_{0}(t, s)=1-|D(t-s)|_{1}(1+o(1)), \quad|t-s| \rightarrow 0,
$$

где $D=\frac{(\lambda+1)^{2}}{2 \lambda} I$.

Очевидно, условие (С3) для поля $V(t)$ имеет место. Выполнение условия $(\mathrm{C} 4)$ следует из вида (4.3) ковариационной функции.

Итак, для поля $V(t)$ справедливы условия теоремы 2.2 при $k=2, \beta=2$, $\alpha_{1}=\alpha_{2}=1$. Применение этой теоремы с учетом (4.7), (4.12), (4.14) позволяет заключить, что при $u \rightarrow \infty$ выполнены соотношения

$$
\begin{gathered}
\mathrm{P}\left\{\left\|w_{0}\right\|_{\gamma}>u\right\}=\mathrm{P}\left\{\sup _{t \in T}|V(t)|>u\right\}=2 \mathrm{P}\left\{\sup _{t \in T} V(t)>u\right\}(1+o(1)) \\
=2 \sqrt{2} H_{1} \exp \left\{-\frac{u^{2}}{2 \tau^{2}}\right\}\left(\frac{u}{\tau}\right)^{3} \pi^{-1 / 2} \frac{(\lambda+1)^{4}}{4 \lambda^{2}} J(u)(1+o(1))
\end{gathered}
$$

где

$$
J(u)=\int_{T_{\delta}} \exp \left\{-u^{2} \tau^{-3} K G^{2}(t)\right\} d t(1+o(1)),
$$

$T_{\delta}=\left\{t \in T: K G^{2}(t) \leqslant \delta^{2}(u)\right\}, \delta(u)$ удовлетворяет условиям (2.18).

ЛЕмма 4.2. При $и \rightarrow \infty$ имеет место соотношение

$$
J(u)=2(\lambda+1)^{-1} \sqrt{\pi} K^{-1 / 2} \tau^{3 / 2} u^{-1}(1+o(1)) .
$$

ДокАЗАТЕЛЬСтво. Множество $T_{0}$ в (4.11) состоит из двух непересекающихся симметричных (относительно главной диагонали) множеств. Поэтому интеграл в (4.16) равен сумме двух интегралов:

$$
J(u)=J^{+}(u)+J^{-}(u),
$$

где

$$
\begin{gathered}
J^{+}(u)=\int_{T_{\delta}^{+}} \exp \left\{-u^{2} \tau^{-3} K\left(t_{2}-t_{1}-x_{0}\right)^{2}\right\} d t(1+o(1)) \\
T_{\delta}^{+}=\left\{t \in T:\left|t_{2}-t_{1}-x_{0}\right| \leqslant \delta_{1}(u)\right\} \\
J^{-}(u)=\int_{T_{\delta}^{-}} \exp \left\{-u^{2} \tau^{-3} K\left(t_{1}-t_{2}-x_{0}\right)^{2}\right\} d t(1+o(1)) \\
T_{\delta}^{-}=\left\{t \in T:\left|t_{1}-t_{2}-x_{0}\right| \leqslant \delta_{1}(u)\right\}
\end{gathered}
$$


$\delta_{1}(u)=\delta(u) / \sqrt{K}, x_{0}=\lambda /(\lambda+1)$. Асимптотики обоих интегралов в (4.18) вычисляются аналогично и оказываются равными. Найдем асимптотику первого интеграла.

Имеем

$$
J^{+}(u)=J_{1}^{+}(u)+J_{2}^{+}(u),
$$

где, как легко видеть, согласно теореме Фубини

$$
\begin{aligned}
& J_{1}^{+}(u)=\int_{0}^{1-x_{0}-\delta_{1}} d s_{1} \int_{-\delta_{1}}^{\delta_{1}} \exp \left\{-u^{2} \tau^{-3} K s_{2}^{2}\right\} d s_{2}, \\
& J_{2}^{+}(u)=\int_{1-x_{0}-\delta_{1}}^{1-x_{0}+\delta_{1}} d s_{1} \int_{-\delta_{1}}^{1-s_{1}-x_{0}} \exp \left\{-u^{2} \tau^{-3} K s_{2}^{2}\right\} d s_{2} .
\end{aligned}
$$

Несложный асимптотический анализ с учетом (2.18) приводит к следующим соотношениям, верным при $u \rightarrow \infty$ :

$$
\begin{aligned}
& J_{1}^{+}(u)=\left(1-x_{0}\right) \sqrt{\pi} K^{-1 / 2} \tau^{3 / 2} u^{-1}(1+o(1)), \\
& J_{2}^{+}(u)=o\left(J_{1}^{+}(u)\right) .
\end{aligned}
$$

Из формул (4.19)-(4.21) находим асимптотику интеграла $J^{+}(u)$.

На основе равенства (4.18) получаем формулу (4.17), что доказывает лемму.

Подставляя (4.17) в (4.15) и учитывая формулы (4.8), (4.10), (4.13), получим соотношение (1.7), поскольку $H_{1}=1$. Теорема 1.2 доказана.

\section{Список литературы}

1. Колмогоров A. Н. К теории непрерывных случайных процессов // Теория вероятностей и математическая статистика. М.: Наука, 1986.

2. Лоэв М. Теория вероятностей. М.: ИЛ, 1962.

3. Belayev Yu. K. Continuity and Hölder conditions for sample functions of stationary Gaussian processes // Proc. of the Fourth Berkeley Sympos. on Math. Statistics and Probability. 1961. V. 2. P. 23-33.

4. Крамер Г., Лидбеттер М. Стационарные случайные процессы. М.: Мир, 1969.

5. Ciesielski Z., Kerkyacharian G., Roynette B. Quelques espaces fonctionnels associés à des processus gaussiens // Stud. Math. 1993. V. 107. № 2. P. 171-204.

6. Фаталов В.Р. Асимптотики вероятностей больших уклонений гауссовских полей и их применения в теории статистик Колмогорова-Смирнова // Теория вероятн. и ее примен. 1984. T. 29. № 1 . С. $178-180$.

7. Фаталов В.Р. Асимптотики вероятностей больших уклонений гауссовских полей // Изв. АН Армении. Математика. 1992. Т. 27. №6. С. 59-81.

8. Фаталов В. Р. Асимптотики вероятностей больших уклонений гауссовских полей. Применения // Изв. АН Армении. Математика. 1993. Т. 28. № 5. С. 32-55.

9. Питербарг В. И. Асимптотические методы в теории гауссовских случайных процессов и полей. М.: Изд-во МГУ, 1988.

10. Питербарг В. И., Фаталов В. Р. Метод Лапласа для вероятностных мер в банаховых пространствах // УМН. 1995. Т. 50. №6. С. 57-150.

11. Baldi P., Roynette B. Some exact equivalents for Brownian motion in Hölder norm // Prob. Theory Rel. Fields. 1992. V. 93. № 4. P. 457-484.

12. Baldi P., Ben Arous G., Kerkyacharian G. Large deviations and the Strassen theorem in Hölder norm // Stoch. Proc. Appl. 1992. V. 42. P. 171-180. 
13. Lorang G., Roynette $B$. Un théorème de Schilder pour des fonctionnelles browniennes non régulières // Ann. Inst. H. Poincaré. Probab. et Statist. 1993. V. 29. № 4. P. 513-530.

14. Колмогоров A.H. Спираль Винера и некоторые другие интересные кривые в гильбертовом пространстве // ДАН СССР. 1940. Т. 26. № 2. С. 115-118.

15. Mandelbrot B. B. The fractional geometry of nature. San Francisco: Freeman, 1982.

16. Michna $Z$. On tail probabilities and first passage times for fractional Brownian motion // Math. Methods Oper. Res. 1999. V. 49. № 2. P. 335-354.

17. Pickands J. III. Upcrossing probabilities for stationary Gaussian processes // Trans. Amer. Math. Soc. 1969. V. 145. P. 51-73.

18. Лидбеттер M., Линдәрен $Г$., Ротсен $X$. Экстремумы случайных последовательностей и процессов. М.: Мир, 1989.

19. Shao Q.-M. Bounds and estimators of a basic constant in extreme value theory of Gaussian processes // Statist. Sinica. 1996. V. 6. P. 245-257.

20. Yakir B., Pollak M. A new representation for a renewal-theoretic constant appearing in asymptotic approximations of large deviations // Ann. Appl. Probab. 1998. V. 8. № 3.

21. Фаталов В.P. Асимптотики вероятностей малых уклонений в $L^{2}$-норме для двух классов гауссовских стационарных процессов // Теория вероятн. и ее примен. (в печати).

22. Фаталов В.Р. Константы в асимптотиках вероятностей малых уклонений для гауссовских процессов и полей // УМН. 2003. Т. 58. № 4. С. 89-134.

23. Adler R. J. On excursion sets, tube formulas and maxima of random fields // Ann. Appl. Prob. 2000. V. 10. № 1. P. 1-74.

24. Питербарг В. И., Присяжнюк В. П. Точная асимптотика вероятности большого размаха гауссовского стационарного процесса // Теория вероятн. и ее примен. 1981. Т. 26. № 3. C. $480-495$.

25. Лифшич M. А. Гауссовские случайные функции. Киев: ТВиМС, 1995.

26. Бородин А.Н., Салминен П. Справочник по броуновскому движению. С.-Пб.: Лань, 2000.

27. Ben Arous G., Ledoux M. Grandes déviations de Freidlin-Wentzell en norme hölderienne // Lect. Notes Math. 1994. V. 1583. P. 293-299.

28. Ledoux M. Isoperimetry and Gaussian analysis // Lect. Notes Math. 1996. V. 1648. P. $165-294$.

29. N'zi M. Strassen theorem in Hölder norm for some Brownian functionals // Ann. Math. Blaise Pascal. 1997. V. 4. № 2. P. 49-59.

30. Фаталов B.P. Большие уклонения гауссовских мер в пространствах $l^{p}$ и $L^{p}, p \geqslant 2$ // Теория вероятн. и ее примен. 1996. Т. 41. № 3. С. 682-689.

31. Фаталов В.P. Асимптотики больших уклонений винеровских полей в $L^{p}$-норме, нелинейные уравнения Хаммерштейна и гиперболические краевые задачи высокого порядка // Теория вероятн. и ее примен. 2002. Т. 47. № 4. С. 710-726.

32. Фаталов B.P. Асимптотики больших уклонений гауссовских процессов типа винеровского для $L^{p}$-функционалов, $p>0$, и гипергеометрическая функция // Матем. сб. 2003. T. 194. №3. C. $61-82$. 